\title{
Epidermólisis ampollosa de la unión: implicación oral. A propósito de un caso
}

\section{Junctional epidermolysis bullosa: oral implication. A case report}

\author{
Pipa Vallejo A*, López-Arranz Monje E*, González García M**, Ortiz Mauriz J***, \\ Pipa Muñiz $M^{* * * * *}$
}

\section{RESUMEN}

Se entiende por epidermólisis ampollosa un grupo de dermatosis no inflamatorias, mecano ampollosas, enfermedades clínicamente similares con afectación cutáneo mucosa, que tienen carácter hereditario, en general, y que se caracterizan por una excesiva fragilidad de la piel y mucosas ante pequeños o mínimos traumas mecánicos, dando lugar a la aparición de vesículas, ampollas, erosiones y escaras. Existe una gran variabilidad fenotípica y considerable morbimortalidad.

Palabras clave: Epidermólisis ampollosa, anquiloglosia, hipoplasia esmalte, ampolla oral.

\section{SUMMARY}

Epidermolysis bullosa is the name given to a group of rare genetically determined diseases of de skin and mucosal membranes, characterized by the development of blisters and vesicles in response to minor trauma. Is characterized by extensive phenotypic variability with considerable morbidity and mortality. Epidermolysis bullosa is classified into distinct subtypes depending on the location of blistering within the cutaneous dermoepidermal basement membrane zone.

Key words: Epidermolysis bullosa, ankyloglossia, enamel hypoplasia, oral blister.

Fecha de recepción: 30 de enero de 2009.

Aceptado para publicación: 2 de marzo de 2009.

* Profesor Asociado. Escuela de Odontología. Facultad de Medicina. Universidad de Oviedo.

** Profesor Asociado. Servicio de Cirugía Oral y Maxilofacial del HUCA.

*** Odontóloga. Colaboradora de Honor. Escuela de Odontología. Universidad de Oviedo.

**** Licenciada en Medicina. Universidad de Oviedo.

Pipa Vallejo A, López-Arranz Monje E, González García M, Ortiz Mauriz J, Pipa Muñiz M. Epidermólisis ampollosa de la unión: implicación oral. A propósito de un caso. Av. Odontoestomatol 2010; 26 (2): 81-90.

\section{INTRODUCCIÓN}

El concepto de epidermólisis ampollosa o ampollar (EA) se aplica a un conjunto de enfermedades ampollares genéticamente determinadas que se caracterizan por un aumento de la susceptibilidad cutá- neo mucosa, apareciendo ampollas, costras y cicatrices en la piel y mucosas frente a un mínimo trauma, por la previa separación entre epitelio y tejido conectivo subyacente, al existir una alteración de las distintas proteínas que intervienen en la unión de la epidermis con la dermis (Fig.1). 


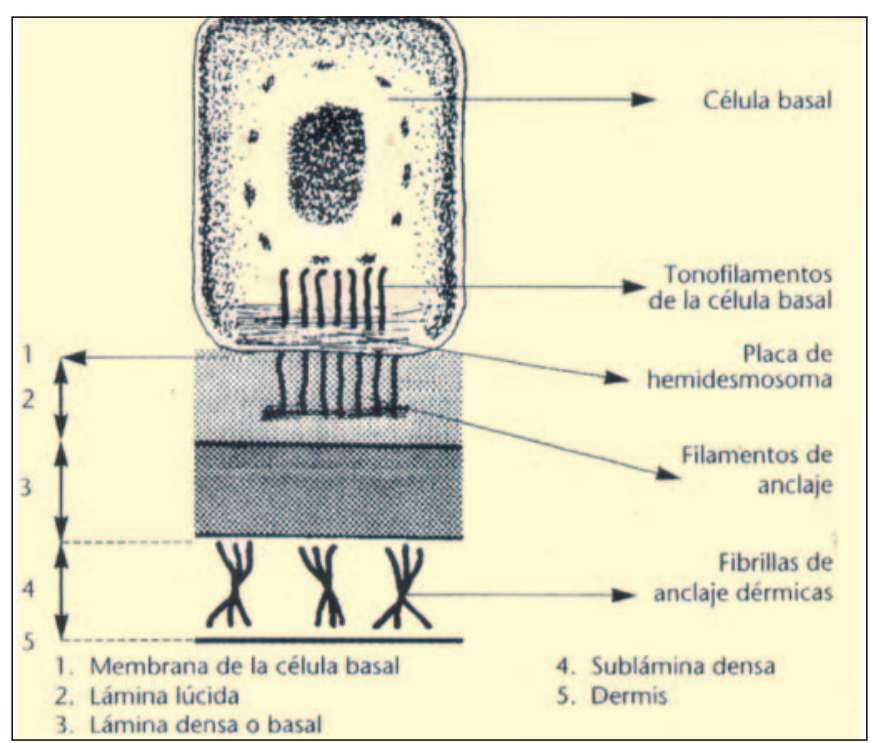

Fig. 1. Estructura de la unión dermo-epidérmica.

Una de las mucosas más frecuentemente afectada es la del tubo digestivo, con retracciones cicatriciales a nivel de esófago dando lugar a disfagia, roturas esofágicas; a nivel bucal, como consecuencia de cicatrices de ampollas y vesículas podemos ver microstomía con dificultad para apertura bucal, anquiloglosia que conlleva acusada limitación de la movilidad de la lengua, pérdida de rugosidades del paladar, así como una obliteración o cierre del vestíbulo oral y depapilación lingual; del tracto genitourinario, con frecuente estenosis uretral, hipertrofia de vejiga; área otorrinolaringológico, con alteraciones a nivel de laringe y conducto auditivo; afectación ocular, con conjuntivitis, blefaritis, úlceras corneales, queratitis. Además de piel y mucosas en las EA puede haber afectación de: uñas, pelo, dientes (por alteraciones en la formación del esmalte), retraso del crecimiento, anemia, neumonía, infecciones, tumores, etc. (1-3).

Existen distintos niveles histológicos en los que se inicia la ampolla o se produce el defecto de la cohesión epitelio-conectiva:

1. Intraepidérmico, separación entre los queratinocitos de la capa basal y membrana basal.

2. Lámina lúcida o junction, separación entre la lámina lúcida y la unión dermoepidérmica. Existe alteración en determinados componentes de los hemidesmosomas, que son rudimentarios y escasos.
3. A nivel de la dermis, debajo de la membrana basal-sublámina densa.

Tradicionalmente se hacían tres grupos de EA que siguen un patrón hereditario y un grupo de epidermólisis adquirida.

Recientemente se ha propuesto un cuarto grupo de patrón hereditario: la variante hemidesmosomal, en el que la separación de los tejidos es en la interfase célula basal y lámina lúcida, a nivel de los hemidesmosomas (4-7).

Las formas heredadas se pueden clasificar en:

- Simple: mutaciones en los genes que codifican queratina 5 y queratina 14 (localizados en 17q12q21,12q13). Existe una rara variante en que se ha demostrado un defecto del gen que codifica la plectina y que cursa también con distrofia muscular. Herencia autosómica dominante en la mayoría de los casos. Hay dos formas recesivas (la generalizada y la letal) y una ligada al sexo, la de Mendes da Costa (8).

- De la unión o junctional (EAJ): mutaciones en los genes LAMA3, locus 18q11.2, LAMB3, localizado en 1q32, LAMC2, localizado en 1q25-q31, relacionados con la codificación de la laminita 5 . La herencia es autosómica recesiva.

- Hemidesmosomal: se han descrito defectos en el gen del colágeno tipo XVII, (COL 17 A1, localizado en 10q24.3, que codifica el antígeno BP180) y una variante que cursa con atresia pilórica y cuyas mutaciones responsables asientan en los genes que codifican la integrina $\alpha 6 \beta 4$, locus $17 q 11$-qter.

- Distrófica: mutaciones en el gen del colágeno tipo VII, componente mayoritario de las fibrillas de anclaje (COL 7A1, locus 3p21.1). Herencia autosómica, dominante o recesiva $(9,10)$.

\section{OBSERVACIÓN CLÍNICA}

En la Facultad de Odontología es vista una niña de cinco años remitida por el servicio de Genética del HUCA para su abordaje bucal dado que presenta un deterioro dental muy acusado. A la exploración se observan numerosas caries, así como defectos en la estructura coronal de dientes temporales (Figs. 2 y 3 ). 


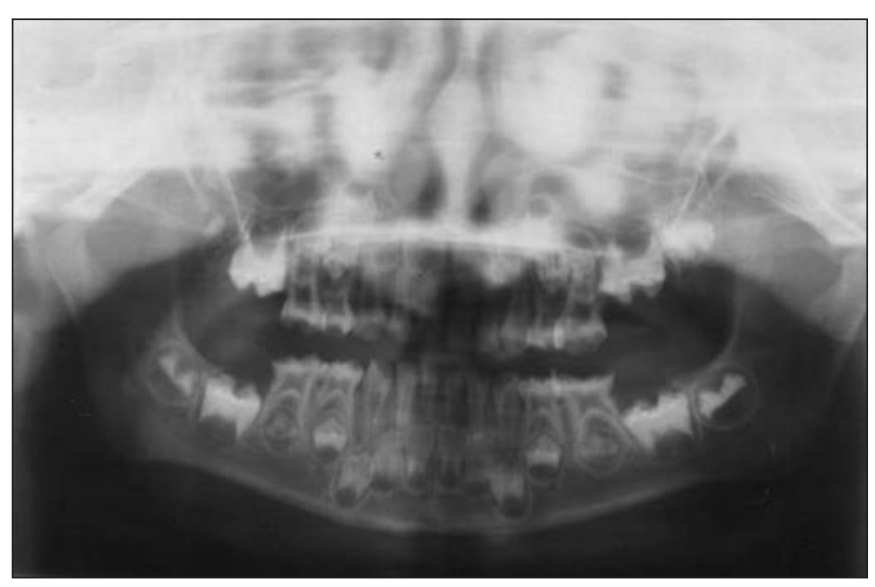

Fig. 2

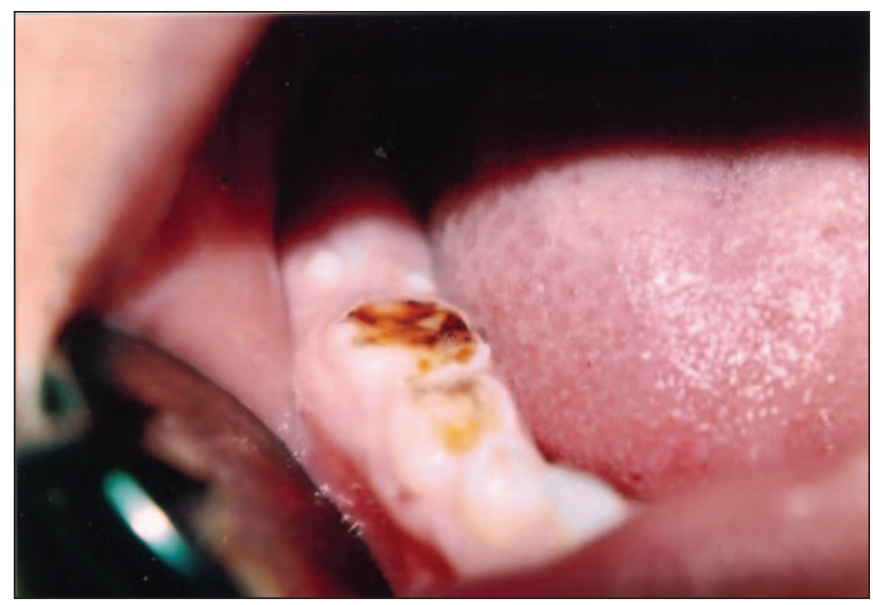

Fig. 3

Presenta numerosas ampollas en manos, pies, brazos, cara, cicatrices de anteriores vesículas, etc. El estudio anatomopatológico diagnostica EA, manifestada neonatalmente y expresada en grado importante aunque no severo: no muestra estenosis esofágica, ni fusión digital, manteniendo en parte las uñas y ampollas que cicatrizan sin graves distrofias. Así mismo presenta hipoacusia neurosensorial. Alopecia difusa (Figs. 4 y 5).

Normalidad en las articulaciones, ampollas generalizadas serohemorrágicas, tensas, que se rompen y se cubren de costra, en cara, mano y pies. No se observan quistes de millium, ni queratodermia palmoplantar, con imágenes cicatriciales de cambio de color en la piel

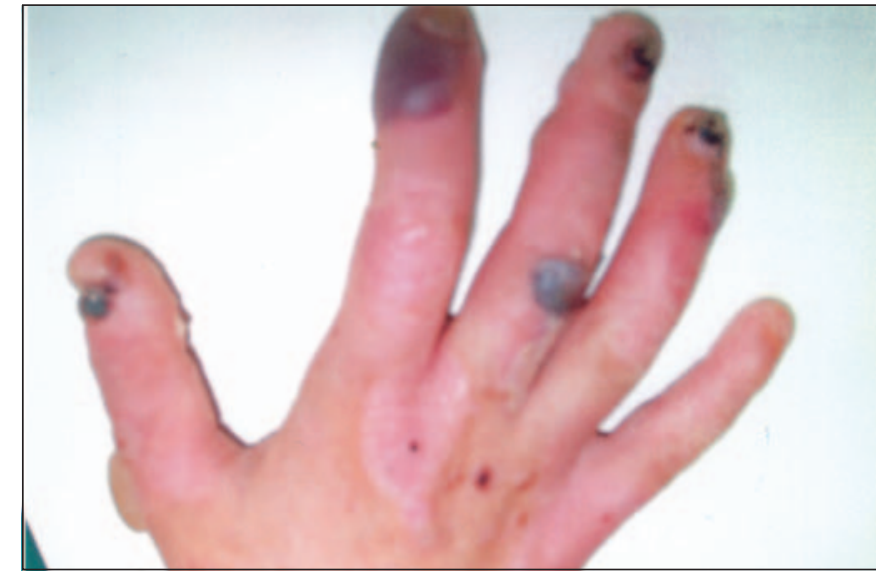

Fig. 4

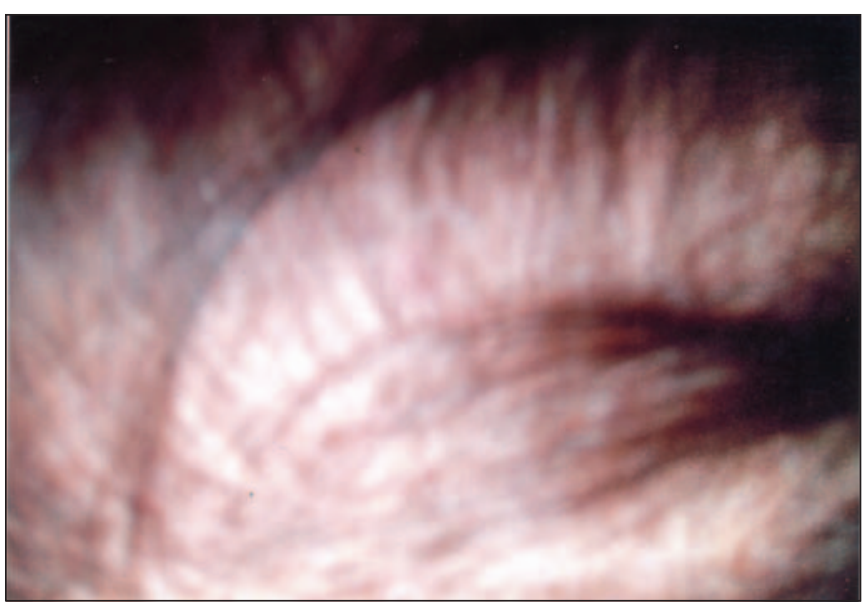

Fig. 5

Embarazo normal, parto a término eutócico. Vacunas controladas.

El diagnóstico ORL demuestra una cocleopatía bilateral con hipoacusia perceptiva bilateral más acusada en OI.

\section{Antecedentes familiares}

Su padre tiene diabetes mellitus. Otras dos hermanas y un hermano así como sus padres son normooyentes y sin afectación cutánea.

Tiene una hermana mayor con el mismo proceso en el mismo momento de nacimiento por pretérmino 
de 35 semanas con desprendimiento de piel y mucosas peri y oralmente. No refieren historia de enfermedades de la piel excepto la abuela materna, que tuvo una niña con EA.

Sus padres son primos carnales y su abuela materna es prima del abuelo paterno. Varios tíos sufrieron la enfermedad ampollosa muriendo cuatro de ellos al nacer.

La biopsia neonatal de la piel determina "marcada ausencia de hemidesmosomas", con el diagnóstico anatomopatológico final de EA juntural atrófica benigna generalizada.

\section{DISCUSIÓN}

En la EA simple, las ampollas son serosas, hemorrágicas, pared gruesa, de presentación más frecuente en manos, pies, cuello y con una afectación oral del $2 \%$. Su incidencia va desde el 1: 50.000 al 1: 100.000 en recién nacidos. Es la forma más frecuente y benigna de EA (11-14).

En la EA de la unión el defecto se encuentra en los filamentos de anclaje de la lámina lúcida, los desmosomas de las células basales epiteliales están disminuidos en numero y tamaño, por lo que se cree que el defecto en su estructura es la causa de la formación de las vesículas. Es la forma más grave de EA.

Existe una variante EAJ letalis de Herlitz que se manifiesta al nacer, con una mortalidad muy elevada en los primeros años de vida y una aparición generalizada y difusa de ampollas, que sin dejar cicatriz curan, salvo infección, pudiendo dejar una piel atrófica, especialmente en manos y pies, en zonas de recurrencia vesicular. Muy característico es la aparición de placas de tejido de granulación exuberante, frecuentes sobretodo alrededor de boca y nariz y que puede desfigurar al niño notablemente. La afección ungueal repetida da lugar a onicodistrofia. Si no se produce la muerte por sobreinfección, los pacientes muestran un retraso pondoestatural importante y anemia intensa. El curso de la enfermedad suele ser tórpido y crónico con periodos de mejorías y exacerbaciones.
A nivel bucal con frecuencia se localiza la lesión entre paladar blando y duro, pudiendo extenderse al esófago con la consiguiente dificultad para la deglución. Estudios sobre la composición de los dientes ponen en evidencia la alteración y desorganización en el epitelio externo del germen y de su mineralización posterior, siendo dientes hipoplásicos, pequeños, decolorados, deformados y muy susceptibles a desarrollar caries con rapidez. Es característica también la afectación laríngea que se evidencia con el clásico lloro afónico y estridor con el consiguiente riesgo de asfixia (15-17).

Hay una EAJ atrófica generalizada benigna, tipo no Herlitz, o mitis, que también se manifiesta en el nacimiento y que en los primeros meses de vida es difícil de diferenciar de la letalis. Predomina la afectación de las extremidades aunque también se pueden ver ampollas en tronco, cuero cabelludo y cara. La intensidad es menor y el proceso tiende a mejorar con la edad y el retraso pondoestatural y la anemia no son tan severas. Puede haber áreas de alopecia atrófica así como ausencia de vello púbico y axilar. Las ampollas, que normalmente son serosanguinolentas, se exacerban en número con el calor ambiental.

El curso de la enfermedad poco a poco se va haciendo menos grave con el paso de los años. Las ampollas tienden a localizarse en zonas acras, en relación con traumatismos.

En la EA distrófica las ampollas son subepidérmicas. Su incidencia se calcula en un 2 por 100.000. Con frecuencia se afecta mucosa oral y presentan caries que son debidas, más a limitación de los movimientos para el cepillado, que a hipoplasia del esmalte propiamente (18-21).

En las heredadas con carácter recesivo existe un aumento del riesgo de padecer carcinoma espinocelular a edades tempranas (22-24).

Aunque en un principio el diagnóstico de EA no es difícil, sí resulta dramáticamente complejo determinar con precisión el tipo básico y el subtipo al que pertenece el paciente. Lo difícil a veces del diagnóstico de EA es establecer el grupo de epidermólisis lo cual requiere una evaluación clínica, genéti- 
ca, inmunológica, microscópica y de laboratorio Hay hasta 30 subtipos de EA que pueden clasificarse según sus características histológicas y microscópicas.

El diagnóstico se hace en un principio con la historia clínica, no obstante, es necesaria una biopsia de las vesículas y del tejido normal adyacente para confirmarlo.

Clínicamente se debe tener en cuenta: la edad de aparición de las vesículas o ampollas, distribución, severidad, exacerbación estival, evolución hacia distrofia, atrofia, formaciones miliares, cicatrices...

Es interesante la información genética cuando existe en la familia un caso con una expresión autosómica dominante; por otro lado la aparición espontánea en una persona de un cuadro ampollar sin antecedentes previos en la familia evidencia no sólo un caso de herencia autosómica recesiva, sino que haya ocurrido una mutación genética (25).

Para el diagnóstico de aproximación es muy útil el estudio histopatológico. En ocasiones la microscopia óptica muestra una ampolla epidérmica y subepidérmica, aunque la microscopia electrónica es determinante para dejar bien clara la localización de la ampolla en los tipos de EAJ, distróficas y adquiridas. Permite objetivar alteraciones de los monofilamentos, de las de los desmosomas, defectos de las fibrillas y de los hemidesmosomas según el distinto tipo de epidermólisis. Este estudio histopatológico se complementa con microscopia convencional, análisis histoquímico y estudio molecular de los genes de las proteínas involucradas.

Para el diagnóstico inmunológico se utilizan anticuerpos de colágena tipo IV, laminina y antígeno de penfigoide bulloso, que forman parte de la lámina densa, la primera y de la lámina basal, los otros. Con técnicas de inmunofluorescencia podemos ver la situación de los marcadores: en la EA simple se dibuja el suelo de la ampolla, en la EAJ los marcadores frente a colágena tipo IV y laminina se ven en el suelo de la ampolla mientras el antígeno del pénfigo lo hace en el techo de la ampolla y en la EA distrófica los marcadores aparecen en el suelo de la ampolla.
El estudio ultraestructural permite definir el tipo y variante de EA, pero debe ser complementado con la información obtenida con las otras técnicas.

El diagnóstico prenatal está elaborado a partir de queratina 5 y 14 en el tipo de EA simple dominante, colágena IV en EA distrófica dominante y en anticuerpos frente a niceína en la EA de la unión recesiva (26).

Desde el punto de diagnóstico diferencial se suele plantear con otros procesos que cursan con ampollas como el pénfigo neonatal, penfigoide gestationis, aplasia cutis, eritema multiforme, psoriasis, herpes simple congénito, varicela, incontinencia pigmentaria, impétigo ampolloso y eritrodermia ictiosiforme ampollosa (27).

Son numerosos los problemas que presentan los pacientes afectados de EA y aunque no se ha encontrado un tratamiento específico, muchas complicaciones pueden atenuarse con una intervención precoz y en algunas situaciones de formas moderadas hay una sintomatología más escasa y requieren un mínimo tratamiento.

Fundamentalmente el tratamiento de estos pacientes es sintomático, a la vez que se pretende apoyarlos y ayudarlos en esta patología a veces tan compleja.

Este cuidado debe enfocarse sobretodo a la prevención de infecciones, a las que son muy susceptibles este tipo de pacientes, ya que nos complica el manejo, retrasa la cicatrización, prolonga la hospitalización y es causa de frecuentes septicemias para lo que es conveniente proteger la piel y en determinados casos las mucosas, de posibles traumas y de manera especial de la fricción. Muy importante es el manejo de las heridas que puedan sufrir, evitando en primer lugar que sobrevenga la infección para lo cual interesa una buena higiene, limpiando suavemente la piel, una buena protección con vendajes absorbentes no adherentes y pomadas antibióticas tópicas a base de mupirocina o ácido fusídico. Es preferible realizar cultivos periódicos y no utilizar siempre el mismo antibiótico par evitar seleccionar cepas resistentes.

Es muy importante prestar atención a la fricción que se pueda ejercer en estos pacientes ya que son más 
sensibles a ella que a la presión, por lo que se debe ser extraordinariamente cuidadoso en el manejo de estas personas.

Es interesante mantener un ambiente fresco, sin calor, lubricar la piel para reducir el roce, evitar comidas o alimentos que puedan ser duros, con alto contenido de ácidos como naranjas, tomates, etc..

Conviene aconsejar e instruir sobre la punción de las ampollas con aguja estéril y posterior compresión para evitar su posible extensión hacia los lados.

Prestar especial consideración a las deficiencias nutritivas que pueden presentar ante la dificultad para la ingesta muy frecuente en este tipo de patologías; la reparación casi constante de las ampollas que conlleva un elevado consumo calórico-protéico, que en algunos casos más graves presentan estos pacientes y que se alimentan con dietas blandas o semisólidas, por la dificultad para digerir alimentos por su afectación esofágica, con frecuencia ricas en $\mathrm{H}$ de $\mathrm{C}$, lo cual unido a la dificultad física para mantener una correcta higiene oral, y a anomalías y defectos del esmalte que suelen presentar hace que sean muy habituales las caries en estos pacientes afectos de EA (28).

Existe en estos pacientes mayor prevalencia de caries, placa dental y de gingivitis lo cual puede ensombrecer y desestabilizar el ya de por sí frágil equilibrio de la cavidad bucal (29-31). No existe en cambio prevalencia significativa de c. albicans, l. casei o s. mutans (32), ni alteración en la secreción de saliva, por lo que la prevalencia de caries en estos pacientes estaría más relacionada con los defectos del esmalte, de los tejidos duros, deficiente higiene o con la dieta (33).

Si hay ampollas orales es aconsejable la alimentación con papillas e insistir en el especial cuidado en el aporte en vitaminas, oligoelementos y hierro por encima de lo normal en estos pacientes (34). A pesar de todo en las formas más graves e invalidantes, debido a la inmovilización, se observa cierto grado de raquitismo latente (35).

Es recomendable instaurar de forma precoz medidas de prevención para reforzar la resistencia del esmalte y así podamos obviar en el futuro tratamientos restauradores complejos en pacientes que ya en muchas ocasiones son difíciles de abordar con operatoria dental convencional (36-38).

Para ello seria conveniente insistir en la higiene oral y todo lo que ello conlleva en cuanto a cepillado, uso de seda dental, insistir en la dieta adecuada en estos pacientes en ocasiones tan susceptibles, evitando productos azucarados muy elaborados, teniendo en cuenta consistencia y adhesividad de los mismos, frecuencia de ingestión, evitando comidas entre horas, que predisponen a la caries, evitar el acúmulo de placa, cálculo, que pueden dar lugar a enfermedad periodontal, insistir en un buen cepillado de dientes, visitas periódicas al dentista, aplicaciones tópicas de flúor, colutorios fluorados, tartrectomías, valorar la conveniencia del uso de selladores de fosas y fisuras de fácil aplicación, e incluso la posibilidad de colocación de coronas de acero preformadas, etc., para un mantenimiento de la cavidad bucal en las mejores condiciones posibles $(39,40)$.

A veces esta higiene oral no es fácil realizarla debido a secuelas cicatriciales en las manos que pueden dar lugar a sindactilias con manos en garra o en manopla o incluso muñones que va a ser una limitación muy acusada a la hora de realizar un correcto cepillado. En estos casos seria conveniente la ayuda por otras personas o la utilización de cepillos dentales adaptados al caso.

Algunos estudios hablan de la efectividad del Sucralfate (útil también en la disminución del dolor y del número de ampollas), en la reducción de la placa dental y de la inflamación y sangrado gingival en unos altos porcentajes, añadido a una buena higiene oral y una dieta alimenticia apropiada (41).

La operatoria dental convencional (obturaciones, exodoncias, RAR), se puede realizar con anestésico tipo lidocaína y con adrenalina al 1:100.000, y se inyecta e infiltra profunda y lentamente dentro de los tejidos para prevenir separaciones mecánicas de los mismos y la aparición de ampollas (42).

Se debe tener un delicado manejo de la mucosa bucal, no habiendo habitualmente problemas especiales en su manipulación. 
Se utilizan idénticos utensilios y materiales que en los demás pacientes para la operatoria dental, insistiendo en un manejo suave, no traumático, delicado, de los tejidos y mucosa oral. A veces durante la manipulación son inevitables las ulceraciones, aunque pueden ser limitadas utilizando una generosa lubricación de los instrumentos intraorales con una crema de hidrocortisona, triamcinolona o pomada de petróleo. En dicha operatoria son preferibles los instrumentos de retracción que tienen forma ancha por que distribuye más la fuerza aplicada sobre la mucosa oral, siendo interesante el uso de puntas de aspiración pequeñas, redondeadas y de plástico en orden a minimizar el daño tisular y disipar el calor que puede ocurrir con instrumentos metálicos grandes. El material de impresión debe ser aplicado con esmero y delicadeza, procurando no realizar fuerzas de fricción o de tracción que son las que pueden producir desgarros mucosos con más facilidad que las fuerzas de compresión o de presión (43).

Es conveniente revisar con relativa frecuencia, aproximadamente cada 3-6 meses, a estos pacientes con especial mención en la higiene oral y en la dieta dado que estos pacientes con una patología dermatológica tan compleja, en ocasiones, están fundamentalmente más pendientes de su afectación dérmica que es en definitiva lo que más les preocupa.

Se abre un rayo de esperanza en el tratamiento con los últimos avances tanto con trasplante de médula ósea, que produce una mejoría en la cicatrización de las heridas, como con la terapia génica, utilizando células troncales de la epidermis modificadas o el empleo de fibroblastos tópicos para inducir la síntesis de colágeno (44).

\section{BIBLIOGRAFÍA}

1. Valle MM. Enfermedades ampollosas. En Lázaro P. Dermatología. Texto y Atlas. Meditécnica, S.A. Madrid 2003.

2. Brooks JK, Bare LC, Davidson J, Taylor LS, Wright JT. Junctional epidermolysis bullosa associated with hypoplastic enamel and pervasive failure of tooth eruption: oral rehabilitation with of an overdenture. Oral Surg Oral Med Oral Pathol Oral Radiol Endod 2008;105:e24-e28.

3. Marinkovich MP. Update on inherited bullous dermatoses. Dermatol Clin 1999;17:473-85.

4. Uitto J, Richard G. Progress in epidermolysis bullosa: from eponyms to molecular genetic classification. Clin Dermatol 2005;23:33-40.

5. Varki R, Sadowski S, Pfendner E, Uitto J. Epidermolysis bullosa.I. Molecular genetics of the junctional and hemidesmosal variants. J Med Genet 2006;43:641-52.

6. Varki R, Sadowski S, Uitto J, Pfendner E. Epidermolysis bullosa.II. Type VII collagen mutations and phenotype-genotype correlations in the dystrophic subtypes. J Med Genet 2007;44:181:92.

7. Fine JD, Eady RAJ, Bauer EA y col. Revised classification system for inherited epidermolysis bullosa: Report of the Second International Consensus Meeting on diagnosis and classification of epidermolysis bullosa. J Am Acad Dermatol 2000;42:1051-66.

8. Smith FJD. The molecular genetics of keratin disorders. Am J Clin Dermatol 2003;4:347-64.

9. Stavropoulos F, Abramowicz S. Management of the oral surgery patient diagnosed with epidermolyisi bullosa: report of 3 cases and review of the literature. J Oral Maxilofac Surg 2008;66: 554-9.

10. Mizrachi-Koren $M$, Shemer S, Indelman $M$ y col. Homozygosity mapping as a screening tool for the molecular diagnosis of hereditary skin diseases in consanguineous populations. J Am Acad Dermatol 2006;55:393-401.

11. Parodi EM, Garcia MI, Gilsanz F. Implicaciones anestésicas de la epidermolisis ampollosa. Act Anest Reanim 2004;14:33-9.

12. Iohom G, Lyons B. Anaesthesia for children with epidermolyisis bullosa: a review of 20 years experience. Eur J Anaesthesiol 2001;18:745-54. 
13. Müller FB, Küster W, Kerstin W y col. Novel and recurrent mutation in keratin KRT5 and KRT14 genes in epidermolysis bullosa simplex: implications for disease phenotype and keratin filament assembly. Hum Mutat 2006;27:719-20.

14. Presland RB, Jurevic RJ. Making sense of the epithelial barrier: what molecular biology and genetics tell us about the functions of oral mucosal and epidermal tissues. J Dent Educ 2002; 66:564-74.

15. Garcia-Pola MJ, Garcia JM, Pipa A. Epidermolisis ampollar. En Bascones A, Seoane JM, Aguado A, Suárez JM. Cáncer y Precáncer Oral. Ediciones Avances. Madrid 2003.

16. Momeni A, Pieper K. Junctional epidermolysis bullosa: a case report. Int J Paediatr Dent 2005; 15:146-50.

17. Herod J, Denyer J, Goldman A, Howard R. Epidermolysis bullosa in children: pathophysiology, anaesthesia and pain management. Paediatr Anaesth 2002;12:388-97.

18. SawamuraD, Niizeki H, Miyagawa S, Shinkuma S, Shimizu H. A novel indel COL7A1 mutation 8068del17insGA causes dominant dystrophic epidermolysis bullosa. Br J Dermatol 2006;154: 995-7.

19. Fewtrell MS, Allgrove J, Gordon I y col. Bone mineralization in children with epidermolysis bullosa. Br J Dermatol 2006;154:959-62.

20. Horn HM, Tidman MJ. The clinical spectrum of dystrophic epidermolysis bullosa. Br J Dermatol 2002;146:267-74.

21. Liversidge HM, Kosmidou A, Hector MP, Roberts GJ. Epidermolysis bullosa and dental developmental age. Int J Paediatr Dent 2005:15: 335-341.

22. Conde-Taboada A, Torre C, Flórez A y col. Epidermolisis ampollosa distrófica pruriginosa con lesiones albopapuloides. Med Cutan Iber Lat Am 2006;34:169-72.
23. Ayman T, Yerebakan O, Ciftcioglu MA, Alpsoy E. A 13-year-old girl with recessive dystrophic epidermolysis bullosa presenting with squamous cell carcinoma. Pediatr Dermatol 2002;19:4368.

24. Christiano AM, Crollick J, Pincus S, Uitto J. Squamous cell carcinoma in a family with dominant dystrophic epidermolisis bullosa: a molecular genetic study. Exp Dermatol 1999;8: 146-52.

25. Cordero C, González S, Castillo C y col. Epidermólisis bulosa distrófica recesiva. Caso clínico. Rev Méd Chile 2004;132:614-8.

26. Marinkovich MP, Meneguzzi G, Burgeson RE. Prenatal diagnosis of Herlitz junctional epidermolisis bullosa by amniocentesis. Prenat Diagn 1995; 15:1027-34.

27. Valdivielso M, Hernanz JM. Epidermólisis ampollosa simple hereditaria. Acta Pediatr Esp 2008; 66:25-6.

28. Kostara A, Roberts GJ, Gelbier M. Dental maturity in children with dystrophic epidermolysis bullosa. Pediatr Dent 2000;22:385-8.

29. Wright JT, Fine JD, Johnson L. Dental caries risk in hereditary epidermolysis bullosa. Pediatr Dent 1994;16:427-32.

30. Birge K. Nutrition management of patients with epidermolysis bullosa. J Am Diet Assoc 1995; 95:575-9.

31. Shuler CF. Inherited risks for susceptibility to dental caries. J Dent Educ 2001;65:1038-45.

32. Harris JC, Bryan RA, Lucas VS, Roberts GJ. Dental disease and caries related microflora in children with dystrophic eepidermolysis bullosa. Pediatr Dent 2001;23:438-443.

33. Wright JT, Childers NK, Evans Kl, Johnson LB, Fine JD. Salivary function of persons with hereditary epidermolysis bullosa. Oral Surg Oral Med Oral Pathol 1991;71:553-9. 
34. Sidbury R, Paller AM. Dermatologic clues to inherited diseases. Pediatr Clin N Am 2000;47: 826-39.

35. Reyes M, Cattani A, Gajardo H y col. Bone metabolism in children with epidermolysis bullosa. J Pediatr 2002;140:467-9

36. Peñarrocha-Diago M, Serrano C, Sanchís JM, Silvestre FJ, Bagán JV. Placement of endosseous implants in patient with oral epidermolysis bullosa. Oral Surg Oral Med Oral Pathol Oral Radiol Endod 2000;90:587-90.

37. Serrano-Martinez MC, Bagan JV, Silvestre FJ, Viguer MT. Oral lesions in recesive dystrophic epidermolysis bullosa. Oral Dis 2003;9:264-8.

38. Lee H, Mardini MA, Ercoli C, Smith MN. Oral rehabilitation of a completely edentulous epidermolysis bullosa patient with an implantsupported prosthesis: a clinical report. J Prosthet Dent 2007;97:65-9.

39. Serrano C, Silvestre FJ, Bagán JV, Peñarrocha M, Alió JJ. Epidermólisis hereditaria: a propósito del manejo odontológico de tres casos. Med Oral 2001;6:48-56.

40. Pipa A, González M, López-Arranz E, Fernández J. Incontinencia Pigmentaria. Consideraciones odontoestomatológicas: Profilaxis y terapéutica. Av Odontoestomatol 2005;21:211-5.

41. Marini I, Vecchiet F. Sucralfate: a help during oral management in patients with epidermolysis bullosa. J Periodontol 2001;72:691-5.

42. Costa F, De Nova MJ, Albajara L, Beltri P. Sedación en Odontopediatria. Aspectos farmacológicos. Rev Eur Odontoestomatol 1997;4:259-70.

43. Brain JH, Paul BF, Assad DA. Periodontal plastic surgery in a dystrophic epidermolysis bullosa patient: Review and case report. J Periodontol 1999;70:1392.

44 Simón C. Epidermólisis ampollosa: Las "alas de mariposa" que no pueden volar. http://www. diariomedico. com/edicion/diariomedico/especialidades/ dermatologia/es/desarrollo/1198110.html (consultado el 20/01/09).

\section{CORRESPONDENCIA}

Adolfo Pipa Vallejo

Escuela de Odontología

Catedrático José Serrano, s/n

33006 Oviedo

Correo electrónico: pipaadolfo@uniovi.es 